\title{
Possible Observation of Phase Transitions in Two-Nucleon Systems
}

\author{
Boris Kostenko* \\ JINR, Dubna, Russia \\ E-mail: bkostenko@jinr.ru \\ Jan Pribiš \\ Technical University, Košice, Slovakia \\ E-mail: jan.pribis@tuke.sk
}

A simple condition of deep cooling for observation of phase transitions in compressed fewnucleon systems was recently proposed. Here we have checked it using data obtained in the first physical experiment with accelerated nuclei at JINR synchrophasotron. Our study was inspired by a remark made by A.M. Baldin et al that one of peaks in observed double differential crosssection may arise due to an "excited state of deuterium nucleus". We have established that one of the peaks in the cross-section corresponds indeed to the dibaryon reported by WASA-at-COSY Collaboration. Another peak in the same region may be explained by interference of several usual baryon resonances.

Even a more amazing fact has been established in a kinematical regions which were considered till now as a contribution of elastic deuteron-deuteron and nucleon(inside deuteron)-deuteron scattering. More careful calculations have shown that it is not the case. Trying to understand the nature of these peaks we looked over many dibaryons reported by different experimental groups and found that they may be excellently explained in terms of light dibaryons with equidistant mass spectrum observed by Yu.A. Troyan in a very different experiment. A natural explanation of these dibaryons may be given on basis of generalized coherent states discovered by A. Perelomov. These light dibaryons can be an experimental evidence for the pion Bose-Einstein condensate appearance in compressed and cooled nucleon systems. The condensate emerges due to a nonperturbative effect described by Bogoliubov's transformation which produces a pion state beyond the range of the Fock space. It should be also noted that this state of pion field has a mathematical and physical prototype in quantum optics, known there as the squeezed vacuum.

Further experimental studies based on modern experimental facilities and more abundant statistics are necessary to verify our conclusions.

XXII International Baldin Seminar on High Energy Physics Problems

September 15-20, 2014

JINR, Dubna, Russia

${ }^{*}$ Speaker. 


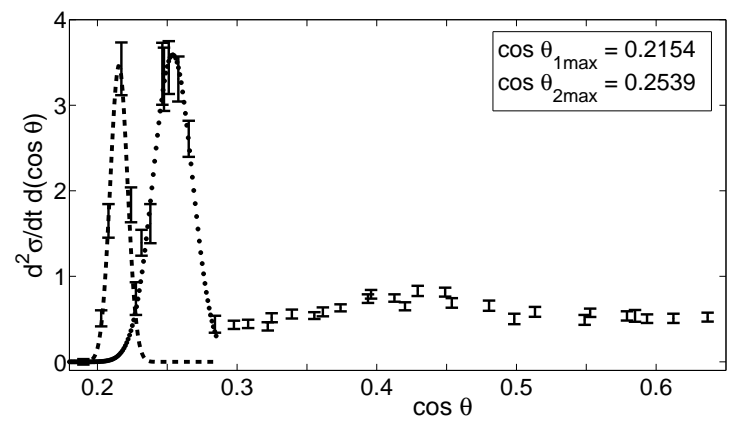

Figure 1: Double differential cross-sections, in $\mathrm{mb} \cdot \mathrm{c}^{2} / \mathrm{GeV}^{2}$, of the $\mathrm{D}+\mathrm{D} \rightarrow \mathrm{M}_{X}+\mathrm{D}$ reaction against cosine of the target nucleus scattering angle [3]. The dashed and dotted curves correspond to approximations of the first two peaks by the Gaussian functions which maxima positions are given in the insertion.

\section{Introduction}

Recently a proposal of QCD investigation at high density and low temperature complementary to the high-energy heavy nuclear collisions was suggested [1, 2]. The proposal is based on the fact that a large number of nucleons in the interaction region is not necessary for the phase transition to occur, and only a change of the vacuum state should be initiated by some experimental environment. In particular, observation of multi-baryons(MB) may be a direct evidence of phase transitions in small nucleon systems. Separation of a MB mass from the secondary particle background is feasible if the MB decay width is narrow enough. That requires the excitation energy of produced MB should be low. For this purpose, it is reasonable to select only those experimental events in which the MB creation is accompanied with a high momentum particle, taking away an essential part of the energy from the interaction region - a cooling effect. Another possible way of essential cooling is selecting events with a considerable amount of secondary pions outgoing the interaction region. In this paper, we focus on new developments of this concept and put them in a context with some of older experimental data taken at JINR synchrophasotron [3-5].

An experiment [3] was designed for measurement of the cross-sections of elastic pp-, ND-, and DD-scattering at $8.9 \mathrm{GeV}$ momentum of primary protons and deuterons. Particularly, three peaks were observed in the spectrum of the missing masses of the reaction $\mathrm{D}+\mathrm{D} \rightarrow \mathrm{M}_{X}+\mathrm{D}$ at $t=$ $-0.495 \mathrm{GeV}^{2}$ (see Fig. 1). Till now the first of them corresponding to the most heavy $\mathrm{M}_{X}$ was estimated to cover the elastic DD scattering; the second one was interpreted as a manifestation of the scattering of a projectile deuteron's nucleon by the target deuteron. In regard to the third peak, it was suggested that contributions of an excited state of deuteron (e.g., 6q-bag), or constituent quark scattering may be seen there too. A kinematic manifestation of a baryons $\mathrm{N}^{*}$ with masses in the neighborhood of $1400 \mathrm{MeV}$ was also suggested to be relevant there.

Experimental findings occurred after the paper [3] was written give cause for re-examination of its conclusions. Data from [4, 5] employing 38915 events will play an especially important role in our consideration. We begin with consideration of the third peak. Thereafter problems concerning the first two ones will be discussed. The present paper might be considered as a particular proposal for experimental search of phase transitions in small nucleon systems. 
Table 1: Spin, parity and width of $N^{*}$ included in our PWA. The data are given by Particle Data Group [7].

\begin{tabular}{|c|c|c|c|}
\hline $\mathrm{N}^{*}$ & $S_{N^{*}}$ & $P_{N^{*}}$ & $\Gamma_{N^{*}}, \mathrm{MeV}$ \\
\hline $\mathrm{N}(1440)$ & $1 / 2$ & 1 & 300 \\
$\mathrm{~N}(1520)$ & $3 / 2$ & -1 & 115 \\
$\mathrm{~N}(1535)$ & $1 / 2$ & -1 & 150 \\
\hline
\end{tabular}

\section{The third peak: constituent quark scattering}

Elastic scattering of a constituent quark by the target deuteron may be considered in the framework of a model in which values of momentum and mass of the projectile quark are considered in the form

$$
P_{q}=x P_{1}, \quad M_{q}=x M_{D}
$$

where $x$ is determined from kinematics of the reaction. All kinematic kinematic relations given in this paper can be found as follows. Let us denote by $1+2 \rightarrow 3+4$ a reaction at issue, where the projectile, target and registered particles are designated by 1, 2 and 4, correspondingly, and 3 denotes an object $\mathrm{X}$ which mass should be determined. Two different expressions for the Lorentz invariant Mandelstam variable $u, u=\left(p_{1}-p_{4}\right)^{2}=\left(p_{2}-p_{3}\right)^{2}, p_{i}=\left(E_{i}, \mathbf{P}_{i}\right),\left|\mathbf{P}_{i}\right|=\sqrt{E_{i}^{2}-M_{i}^{2}}$, allow to connect $M_{X}$ and $\cos \theta$. The energy of particle 4 as function of $M_{2}, M_{4}$ and $t$ may be found by making use of a relation $t=\left(p_{2}-p_{4}\right)^{2}$. In addition, $E_{3}=E_{1}+E_{2}-E_{4}$. The most important formulae for $M_{X}$ in different models are given in our paper explicitly as their derivation is rather cumbersome. Because of it, our calculations were assisted by some computer algebra.

The constituent quark model gives for $\cos \theta=0.396$ a value of quark mass,

$$
M_{q}=\frac{-M_{D}^{2} t}{E_{1} t+P_{1} \sqrt{t\left(-4 M_{D}^{2}+t\right)} \cos \theta},
$$

about $0.351 \mathrm{GeV}$. This number contradicts manifestly to estimations of modern quark models: see, e.g., [6] where $M_{q}=0.318 \mathrm{GeV}$. On the other hand, we shall see below that the peak at $\cos \theta=0.396$ corresponds remarkably to the dibaryon found by WASA-at-COSY Collaboration.

\section{Partial-wave analysis (PWA) and $S U(6) \otimes O(3)$ quark spectroscopy}

Now let us turn to a possible contribution of $N+D \rightarrow N^{*}+D$ reactions to the third peak. Isotopic spin conservation constrains isospin of $N^{*}$ to be equal to $1 / 2$. Therefore, $\Delta$-baryon excitations of nucleon may be ignored here, and among $N^{*}$ excitations only $\mathrm{N}(1440), \mathrm{N}(1520)$ and $\mathrm{N}(1535)$ are important in the kinematic region under consideration. Main characteristics of the baryon resonances taken into account are shown in Table 1.

Besides spatial parity, $\hat{P}$, conservation, one should respect angular momentum, $\hat{\vec{J}}=\hat{\vec{L}}+\hat{\vec{S}}$, preservation. In $s p$-approximation, appropriate to the hard collisions, only $L=0$ and $L=1$ eigenvalues of orbital momenta can be considered. In these terms, parities of initial and final states may 
be expressed as follows:

$$
P_{i}=P_{N} P_{D}(-1)^{L_{i}}=(-1)^{L_{i}}=P_{f}=P_{N^{*}}(-1)^{L_{f}},
$$

correspondingly.

Further partial-wave analysis may be essentially simplified via application the $S U(6) \otimes O(3)$ description of baryon excitations, suggested by R.H. Dalitz and co-authors [8]. According to it, spin $\vec{S}_{N^{*}}$ of a nucleon resonance $N^{*}$ may be represented as follows:

$$
\vec{S}_{N^{*}}=\vec{S}_{N}+\vec{l}
$$

where $\vec{S}_{N}$ is spin of unexcited nucleon $N$ and $\vec{l}$ is orbital momentum of quarks inside of nucleon. Using (3.1), it is readily seen that for each partial wave, which is characterized by fixed values of $J$ and $P$, a value of parity $P_{N^{*}}$ of nucleon resonance $N^{*}$ determines totally possible behavior of $l$ and $L$ values. For N(1440), one has $P_{N^{*}}=P_{N}=1$ which implies $l=0$, and, subject to (3.1), also $L_{f}=L_{i}$. For $\mathrm{N}(1520)$ and $\mathrm{N}(1535), P_{N^{*}}=-1$; therefore $l=1$. According to (3.2) and Table 1, we can interpret spins of $\mathrm{N}(1520)$ and $\mathrm{N}(1535)$ as two different manners of summation, using ClebschGordan coefficients, of quark orbital momentum $l=1$ and initial spin $S_{N}=1 / 2$ of unexcited nucleon. Parity conservation leads to simultaneous change of $L$ and $l$ values in two possible ways:

$$
L_{i}=1 \rightarrow L_{f}=0, \quad l_{i}=0 \rightarrow l_{f}=1,
$$

and

$$
L_{i}=0 \rightarrow L_{f}=1, \quad l_{i}=0 \rightarrow l_{f}=1 .
$$

In the frame of $S U(6) \otimes O(3)$ spectroscopy, these cases correspond to conservation of eigenvalues of operator $\vec{M}^{2}=(\vec{L}+\vec{l})^{2}$, which are equal to 2 and 0 , accordingly. Operator of total orbital momentum $\vec{M}$ commutes with $\vec{M}^{2}$, and we can develop a more detail picture including account of a direction of $\vec{M}$. Below we consider centrally symmetric interaction conserving the direction of $\vec{M}$. In this case, conservation of total angular and orbital momenta implies preservation of total spin of the system, $\vec{S}=\vec{J}-\vec{M}$, and description tolerates further simplification.

A general expression of the $N+D \rightarrow N^{*}+D$ amplitude linear relative to $S_{N}, S_{D}$ and invariant under time reversal and space rotation or reflection is as follows [9]

$$
\begin{gathered}
T\left(\vec{S}_{N}, \vec{S}_{D}\right)=C_{1}+C_{2}\left(\vec{S}_{N}+\vec{S}_{D}\right) \cdot \vec{v}+C_{3}\left(\vec{S}_{N}-\vec{S}_{D}\right) \cdot \vec{v}+C_{4}\left(\vec{S}_{N} \cdot \vec{v}\right)\left(\vec{S}_{D} \cdot \vec{v}\right)+ \\
C_{5}\left(\vec{S}_{N} \cdot \vec{\lambda}\right)\left(\vec{S}_{D} \cdot \vec{\lambda}\right)+C_{6}\left(\vec{S}_{N} \cdot \vec{\mu}\right)\left(\vec{S}_{D} \cdot \vec{\mu}\right)
\end{gathered}
$$

where

$$
\vec{v}=\frac{\vec{p} \times \vec{p}^{\prime}}{\left|\vec{p} \times \vec{p}^{\prime}\right|}, \quad \vec{\lambda}=\frac{\vec{p}-\vec{p}^{\prime}}{\left|\vec{p}-\vec{p}^{\prime}\right|}, \quad \vec{\mu}=\frac{\vec{p}+\vec{p}^{\prime}}{\left|\vec{p}+\vec{p}^{\prime}\right|}
$$

$\vec{p}$ and $\vec{p}^{\prime}$ are momenta of the ingoing nucleon and outgoing $\mathrm{N}^{*}$. Here $C_{i}$ are scalar functions which may depend only on a scalar $\left(\vec{p} \cdot \vec{p}^{\prime}\right) /|\vec{p}|\left|\vec{p}^{\prime}\right|$ which is in one-to-one correspondence with $\cos \theta$ in Fig. 1. In fact, we should claim $C_{3}=0$, for $\vec{S}_{N}-\vec{S}_{D}$ does not commute with $\left(\vec{S}_{N}+\vec{S}_{D}\right)^{2}$ and the corresponding term breaks conservation of absolute value of total spin. Similarly, it is possible to 
show that $C_{4}=C_{5}=C_{6}=0^{1}$. Because of total spin conservation, a term proportional to $\left(\vec{S}_{N}+\vec{S}_{D}\right)^{2}$ is not included in (3.5) as far as it is proportional to unit operator for any state with total spin fixed, $\vec{S}^{2}=S(S+1)$. Efficiently, it is included in $C_{1}$.

We have seen that parity conservation admits concordant alteration of $L$ and $l$ according to (3.3) and (3.4). From the physical point of view (3.3) corresponds to swapping external orbital momentum in $\mathrm{N}+\mathrm{D}$ system into nucleon, and (3.4) corresponds to excitation of both external, $\vec{L}$, and intranucleonic, $\vec{l}$, momenta. These processes may be described by a nonlocal operator $(\vec{R} \cdot \vec{r})$ included in the interaction amplitude. Here $\vec{R}$ is a polar vector given in the laboratory system, which is directed at center of inertia of $\mathrm{N}+\mathrm{D}$ system, and $\vec{r}$ is a polar vector pointed at center of mass of the nucleon colliding with deuteron. Without loss of generality, we may also suggest $(\vec{R} \cdot \vec{R})=(\vec{r} \cdot \vec{r})=1$. Then $T$-matrix describing production of baryon from Table 1 may be written in the form

$$
T\left(N+D \rightarrow N^{*}+D\right)=A+B\left(\vec{S}_{N}+\vec{S}_{D}\right) \cdot \vec{v}+(\vec{R} \cdot \vec{r})\left[C+D\left(\vec{S}_{N}+\vec{S}_{D}\right) \cdot \vec{v}\right] .
$$

Here $B$ describes spin dependent part of interaction corresponding to $\mathrm{N}(1440)$ production, $C$ and $D$ describe interaction corresponding to $\mathrm{N}(1520)$ and $\mathrm{N}(1535)$. Using an identity

$$
(\vec{R} \cdot \vec{r})=\frac{1}{2}\left(R_{+} r_{-}+R_{-} r_{+}\right)+R_{z} r_{z}
$$

and well-known formulae for $\vec{R}$ and $\vec{r}$ operators [9]

$$
\begin{aligned}
\left\langle L=1, M=0\left|R_{z}\right| L=0, M=0\right\rangle=-i / \sqrt{3}, & \left\langle l=1, m=0\left|r_{z}\right| l=0, m=0\right\rangle=-i / \sqrt{3}, \\
\left\langle L=1, M=-1\left|R_{-}\right| L=0, M=0\right\rangle=-i \sqrt{2 / 3}, & \left\langle l=1, m=+1\left|r_{+}\right| l=0, m=0\right\rangle=+i \sqrt{2 / 3},
\end{aligned}
$$

it is possible to find that amplitudes of the processes (3.3) and (3.4) are equal to 1 and $1 / 3$, accordingly.

\section{Observable particles, cross-section}

In fact, baryon resonances N(1440), N(1520) and N(1535) were not observed directly. They were present in an intermediate state and may be identified only via their decay products. Therefore interference terms corresponding simultaneous propagation of matter through several quantum states with different spins and parities should be taken into account. We take for granted that possible final states tolerating macroscopic recognition may contain $\mathrm{N} \pi, \mathrm{N} \pi \pi$ and $\mathrm{N} \eta$, of course, besides deuteron. For $\mathrm{N}(1440)$ and $\mathrm{N}(1520)$, corresponding decay probabilities can be estimated as $w_{1 \pi} \approx 0.65, w_{2 \pi} \approx 0.35, w_{\eta} \approx 0$; and $w_{1 \pi} \approx 0.5, w_{2 \pi} \approx 0.1, w_{\eta} \approx 0.4$ for $\mathrm{N}(1535)$, see [7].

Baryon resonances leave imprint of their existence only as propagators in total amplitude. For example, a transition $\mathrm{N}+\mathrm{D} \rightarrow \mathrm{N}+\pi+\mathrm{D}$ is described by the following $T$-matrix:

$$
T(N+D \rightarrow N+\pi+D)=\frac{A+B\left(\vec{S}_{N}+\vec{S}_{D}\right) \cdot \vec{v}}{M_{N(1440)}^{2}-M_{X}^{2}-i M_{N(1440)} \Gamma_{N(1440)}} T(N(1440) \rightarrow N+\pi)+
$$

\footnotetext{
${ }^{1}$ For any vector $\vec{n}$ an identity $\left(\vec{S}_{N} \cdot \vec{n}\right)\left(\vec{S}_{D} \cdot \vec{n}\right)=\frac{1}{2}\left((\vec{S} \cdot \vec{n})^{2}-\left(\vec{S}_{N} \cdot \vec{n}\right)^{2}-\left(\vec{S}_{D} \cdot \vec{n}\right)^{2}\right)$ holds true. The term $\left(\vec{S}_{N} \cdot \vec{n}\right)^{2}=$ $1 / 4$ in the parentheses preserves $\vec{S}$, the term $(\vec{S} \cdot \vec{n})^{2}$ commutes with $\vec{S}^{2}$, but does not with $\vec{S}$. This means that it conserves absolute value of total spin and breaks its direction. The term $\left(\vec{S}_{D} \cdot \vec{n}\right)^{2}$ does not maintain a direction of $\vec{S}_{D}$ and therefore a direction of $\vec{S}=\vec{S}_{D}+\vec{S}_{N}$ or even an absolute value of total spin.
} 


$$
\begin{aligned}
& +\left[\frac{f(S, 3 / 2)}{M_{N(1520)}^{2}-M_{X}^{2}-i M_{N(1520)} \Gamma_{N(1520)}} T(N(1520) \rightarrow N+\pi)\right]\left(C+D\left(\vec{S}_{N}+\vec{S}_{D}\right) \cdot \vec{v}\right)+ \\
& +\left[\frac{f(S, 1 / 2)}{M_{N(1535)}^{2}-M_{X}^{2}-i M_{N(1535)} \Gamma_{N(1535)}} T(N(1535) \rightarrow N+\pi)\right]\left(C+D\left(\vec{S}_{N}+\vec{S}_{D}\right) \cdot \vec{v}\right) .
\end{aligned}
$$

Analogous expressions take place for $\mathrm{N}+\mathrm{D} \rightarrow \mathrm{N}+\pi+\pi+\mathrm{D}$ and $\mathrm{N}+\mathrm{D} \rightarrow \mathrm{N}+\eta+\mathrm{D}$ transitions. In (4.1), scalar functions $A, B, C, D$ are the same as in (3.6), and coefficients $f\left(S, N^{*}\right)$ may be found on basis of Clebsch-Gordan coefficients, as it was mentioned in previous section. Following this prescription, one can find

$$
f\left(S, S_{N^{*}}\right)=\sum_{\sigma_{1}= \pm 1 / 2} \sum_{\sigma_{2}=0, \pm 1} \sum_{m=0, \pm 1}\left\langle\frac{1}{2} \sigma_{1} 1 \sigma_{2} \mid S, \sigma_{1}+\sigma_{2}\right\rangle\left\langle\frac{1}{2} \sigma_{1} 1 m \mid S_{N^{*}}, \sigma_{1}+m\right\rangle,
$$

and

$$
f\left(\frac{1}{2}, \frac{1}{2}\right)=2+\sqrt{2}, f\left(\frac{3}{2}, \frac{1}{2}\right)=\frac{2}{3}, f\left(\frac{1}{2}, \frac{3}{2}\right)=0, f\left(\frac{3}{2}, \frac{3}{2}\right)=\frac{4}{3}(\sqrt{2}+\sqrt{3}+\sqrt{6}),
$$

where we adopted notations of Clebsch-Gordan coefficients from [9].

Here we should re-arrange a usual formula for cross-section [10],

$$
\frac{d^{2} \sigma}{d t d M_{X}^{2}}=\frac{\pi}{\lambda^{1 / 2}\left(s, m_{N}^{2}, m_{d}^{2}\right)} \frac{1}{\left(2 S_{N}+1\right)\left(2 S_{D}+1\right)} \sum_{\mathrm{M}_{i}, \mathrm{M}_{f}} \int d \operatorname{Lips}\left(M_{X}^{2}, \text { decay products }\right)\left|T_{\mathrm{M}_{i} \mathrm{M}_{f}}\right|^{2},
$$

where $\mathbf{M}_{i}$ and $\mathrm{M}_{f}$ are spin projections of particles in initial and final states, into terms of our model of the orbital nucleon excitations. To this end, we replace averaging over $\mathbf{M}_{i}$ and summation over $\mathrm{M}_{f}$ by corresponding operation over $\Sigma_{i}$ and $\Sigma_{f}$, which are total spin projections of quarks in initial and final states. For nonpolarized initial states, probabilities of occurrence of $S=1 / 2$ and $S=3 / 2$ are equal to $1 / 3$ and $2 / 3$, accordingly. Taking into account that the contribution of orbital excitations is already included by means of $f\left(S, S_{N^{*}}\right)$, we may write:

$$
\frac{1}{\left(2 S_{N}+1\right)\left(2 S_{D}+1\right)} \sum_{\mathrm{M}_{i}, \mathrm{M}_{f}}\left|T_{\mathrm{M}_{i} \mathrm{M}_{f}}\right|^{2}=\frac{1}{3} \sum_{\Sigma_{f}= \pm 1 / 2} \overline{\left|T_{\Sigma_{i} \Sigma_{f}}\right|^{2}}+\frac{2}{3} \sum_{\Sigma_{f}= \pm 1 / 2, \pm 3 / 2} \overline{\left|T_{\Sigma_{i} \Sigma_{f}}\right|^{2}}
$$

and then transform

$$
\sum_{\Sigma_{f}} \overline{\left|T_{\Sigma_{i} \Sigma_{f}}\right|^{2}}=\sum_{\Sigma_{f}} \overline{T_{\Sigma_{i} \Sigma_{f}} T_{\Sigma_{i} \Sigma_{f}}^{*}}=\sum_{\Sigma_{f}} \overline{T_{\Sigma_{i} \Sigma_{f}} T_{\Sigma_{f} \Sigma_{i}}^{\dagger}}=\overline{\left(T T^{\dagger}\right)}{ }_{\Sigma_{i} \Sigma_{i}} \equiv \frac{1}{2 S+1} \operatorname{Tr}\left(T T^{\dagger}\right) .
$$

Now it is easy to prove a relation

$$
\frac{1}{\left(2 S_{N}+1\right)\left(2 S_{D}+1\right)} \sum_{\mathrm{M}_{i}, \mathrm{M}_{f}}\left|T_{\mathrm{M}_{i} \mathrm{M}_{f}}\right|^{2}=\frac{1}{6} \sum_{S=\frac{1}{2}, \frac{3}{2}} \operatorname{Tr}\left(T(S) T(S)^{\dagger}\right)
$$

which means that values of total spin $S=1 / 2$ and $3 / 2$, as well as all its projections $\Sigma= \pm 1 / 2$ and $\Sigma= \pm 1 / 2, \pm 3 / 2$, correspondingly, give equal contribution to the final result. It should be 
stressed that the sign ${ }^{\dagger}$ of Hermitian conjugation refers to $T$ as to spin operator, and it does not mean transposition of other variables ${ }^{2}$.

Calculations of $\operatorname{Tr}\left(T T^{\dagger}\right)$ may be completed with making use of relations:

$$
\operatorname{Tr}(1)=\left\{\begin{array}{ll}
2, & S=1 / 2, \\
4, & S=3 / 2,
\end{array} \quad \operatorname{Tr}(\vec{S} \cdot \vec{v})^{2}=\left\{\begin{array}{ll}
1 / 2, & S=1 / 2, \\
5, & S=3 / 2,
\end{array} \quad \operatorname{Tr}(\vec{S} \cdot \vec{v})=0 .\right.\right.
$$

Absolute values of the decay amplitudes are fixed in terms of decay widths [10],

$$
\Gamma_{N^{*}, f}=\frac{1}{2 M_{N^{*}}} \int d \operatorname{Lips}\left(M_{X}^{2}, f\right) \sum_{\mathrm{M}_{f}}\left|T\left(N^{*} \rightarrow f\right)\right|^{2},
$$

where subscript $N^{*}$ denotes a particular baryon resonance, $f$ is its decay products. We confine our estimations of interference between different baryon resonances to operations with phase space averaged values. For this purpose, we define ${ }^{3}$

$$
\Gamma_{N^{*}, f}=\frac{\left(2 S_{N^{*}}+1\right)}{2 M_{N^{*}}} \mid \overline{\left.T\left(N^{*} \rightarrow f\right)\right|^{2}} \operatorname{Lips}\left(M_{X}^{2}, f\right),
$$

and substitute ${ }^{4}$

$$
\left(2 S_{N^{*}}+1\right)\left(\overline{\left|T\left(N_{i}^{*} \rightarrow f\right)\right|^{2}}\right)^{1 / 2}\left(\overline{\left|T\left(N_{j}^{*} \rightarrow f\right)\right|^{2}}\right)^{1 / 2} e^{i\left(\bar{\alpha}_{i}-\bar{\alpha}_{j}\right)} \operatorname{Lips}\left(M_{X}^{2}, f\right)=2 \sqrt{M_{i} M_{j} \Gamma_{i} \Gamma_{j}} e^{i\left(\bar{\alpha}_{i}-\bar{\alpha}_{j}\right)}
$$

for

$$
\int d \operatorname{Lips}\left(M_{X}^{2}, f\right) \sum_{\mathrm{M}_{f}} T\left(N_{i}^{*} \rightarrow f\right) T^{*}\left(N_{j}^{*} \rightarrow f\right)
$$

if $M_{X}$ is greater than $N^{*}$ decay threshold and zero otherwise. Here baryon resonances are different, $i \neq j$, and decay particles are the same for the both multipliers under integral sign. Strictly speaking, separate control of spin projections of $N^{*}$ is not kept in mind in our description, but only projection of total spin of quarks in the final state of reaction $\mathrm{N}+\mathrm{D} \rightarrow \mathrm{N}^{*}+\mathrm{D}$. Therefore we should take into account availability of deuteron too and replace $\mathrm{M}_{f}$ with $\Sigma_{f}$ and $S_{N^{*}}$ with $S$ in the previous formulae. Such a treatment may be understood as summation over quark spin projections inside $N^{*}$ and spectator deuteron. Contribution of orbital excitations into spin projection of $N^{*}$ is already included explicitly by means of $f\left(S, S_{N^{*}}\right)$, as it was mentioned above. This new interpretation of spin summation rule is an inevitable corollary of consideration of baryon as a compound system with its own inner structure.

In the accepted approximation, only phases of the decay amplitudes $\bar{\alpha}_{i}$ may be used as adjustable parameters for experimental data matching. In addition, eight real numbers corresponding complex parameters $A, B, C, D$ in (4.1) are brought into play for this purpose. Interference terms corresponding decays of $N^{*}$ via $\eta$ are absent since cross-sections of this channel are negligible

\footnotetext{
${ }^{2}$ This mathematical trick is described in [9] in section devoted to spin-orbit interaction.

${ }^{3}$ Hereafter we retain the overline as notation for averaging over Lorentz-invariant phase space.

${ }^{4}$ Using Cauchy-Bunyakovsky-Schwarz inequality, it may be proven that modulus of the interference terms defined by (4.3) is in the general case greater than the true one. Therefore the role of interference is overestimated in our calculations. Thus, we create an optimum for explanation of experimental data by interference between different nucleon excitations, as far as the resonances have too large widths to explain cross-section without it.
} 
quantities but for one of the resonances under consideration (see values $w_{\eta}$ in beginning of this section). The final formula describing the experimental data may be written in the following form:

$$
\frac{d^{2} \sigma}{d t d M_{X}^{2}}=\frac{\pi}{\lambda^{1 / 2}\left(s, m_{N}^{2}, m_{d}^{2}\right)} \frac{1}{6} \sum_{\substack{S=\frac{1}{2}, \frac{3}{2} \\ f=N \pi, N \eta, N \pi \pi}} \int d \operatorname{Lips}\left(M_{X}^{2}, f\right) \operatorname{Tr}\left(T(S, f) T^{\dagger}(S, f)\right)+E,
$$

where an additional adjustable parameter $E$ describes a contribution of direct pion production near $M_{X}^{2}=1.5 \div 2 \mathrm{GeV}^{2}$.

\section{Some details of numerical calculations}

To reach an optimum in describing the experimental data we minimized total deviation square for 22 experimental points chosen in the third peak region from the theoretical curve. Ten central experimental points were taken with unit weights and six ones on their left and right were scaled with 0.5 significance. MAPLE procedure NLPSolve for local minimum search was used for optimal selection of theoretical parameters. Several series, each containing 20000 different sets of random initial values of parameters, were generated and only 30 percent of them were finished without interruption because of very big number of steps towards a local minimum. Points of the interruptions were considered as local minima too, because they usually correspond to wanderings along valleys. Then the best local minimum was taken for each of the series, and values of objective function corresponding to them were compared. They turned out to be equal within accuracy of 11 decimal digits. All the best local optima have demonstrated that experimental data demand unambiguously:

$$
|A|=0, \quad|C|=0 .
$$

This means that phases $\phi_{A}$ and $\phi_{C}$ of complex numbers $A$ and $C$ have no impact upon objective function. For removal of degeneration, we have fixed $\phi_{A}=\phi_{C}=0$ and introduced condition (5.1) explicitly into minimizing functional. Now the normal mode of NLPSolve performance increased up to 55 percent signalling, nevertheless, that a large degeneration still persisted. Three series of numerical experiments, containing 100, 1000 and 20000 events, with random selections of initial values of the remaining parameters were fulfilled. They showed that parameters $|B|,|D|$ and $E$ are identical in all the cases and are determined with accuracy of 4 and 6 decimal digits already in the series with 100 and 1000 events. However, all phases underwent rather strong changes with growth of statistics, signalling that minimizing functional remains degenerate with respect to them. Thus, the optimization problem does not allow us to determine phases of parameters $A, B, C, D$, $T\left(N_{i}^{*} \rightarrow N+\pi\right)$ and $T\left(N_{i}^{*} \rightarrow N+\pi+\pi\right)$, because many of their sets describe equally well the experimental data. A grade of fidelity of reproduction of the experimental data by this model may be seen in Fig. 2.

We have also fulfilled evaluation of the model parameters using only 10 experimental points taken straight from the fine structure location, trying to enhance an impact of the most important region. It was technically fully regular procedure, as far as we had only 8 independent parameters at that stage. However, an agreement between theory and experiment has not improved even in this case. 


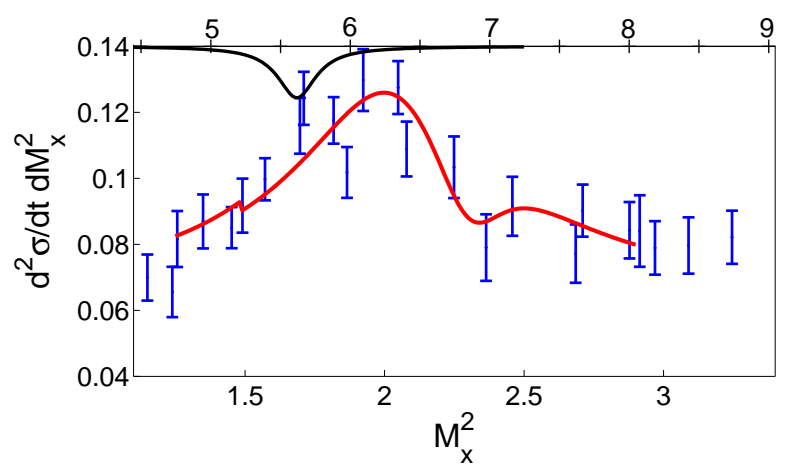

Figure 2: The experimental data (bars) in the range of the third peak and their explanation by the sum of contributions of $\mathrm{N}+\mathrm{D} \rightarrow \mathrm{N}^{*}+\mathrm{D}$ reactions (red line). The lower scale corresponds to the kinematics of reaction $\mathrm{N}+\mathrm{D} \rightarrow \mathrm{N}^{*}+\mathrm{D}$. The top scale describes reactions $\mathrm{D}+\mathrm{D} \rightarrow \mathrm{X}+\mathrm{D}$ which implies the dibaryon production. A possible contribution of a dibaryon at $2.37 \mathrm{GeV}, \Gamma \approx 70 \mathrm{MeV}$ reported by WASA-at-COSY Collaboration $[11]$ into the third region is shown with the overturned black line.

\section{Conclusions concerning the third peak}

Numerical analysis fulfilled within the bounds of our model has revealed two nonobvious properties of hard N-D and D-D scattering. First of all, it was established that experimental data [3] show strong spin dependence of $\mathrm{N}+\mathrm{D} \rightarrow \mathrm{N}^{*}+\mathrm{D}$ transition amplitude, see (3.6) with $A=C=0$. Secondly, comparison of the experimental data and theory shown in Fig. 2 makes an explicit hint about dibaryon production in this kinematic region. Indeed, on the one hand, consideration only usual nucleon excitations cannot explain the fine structure shown in the figure. On the other hand, assumption about presence of a dibaryon at $M_{2 B} \approx 2.38 \mathrm{GeV}, \Gamma_{2 B} \approx 70 \mathrm{MeV}$, seen by WASA-atCOSY Collaboration [11] allows one to explain it very naturally. Isospin conservation predicts certainly that reaction $\mathrm{D}+\mathrm{D} \rightarrow$ dibaryon $+\mathrm{D}$ should yield dibaryon with isospin $I=0$, which also corresponds to the WASA-at-COSY result [11].

To check our conclusions, it would be enough to measure with a good precision production cross-sections of $\mathrm{N}(1440), \mathrm{N}(1520)$ and $\mathrm{N}(1535)$ from $\mathrm{N}+\mathrm{D} \rightarrow \mathrm{N}^{*}+\mathrm{D}$ reactions in appropriate kinematic region, and direct production of pions therein. This allows one to take into account the background. In addition, repeating experiment [3] with higher accuracy is necessary too for unambiguous recognition of dibaryon by its mass and width. Theoretical and experimental study of the phases entering into expression for production amplitude is ineffectual in this respect, so long as resultant cross-section is weakly dependent on them (see previous section). Investigation of decay products of dibaryon will make it possible to identify its spin and parity and compare with $J^{P}=3^{+}$observed in [11].

\section{The first two peaks' puzzle}

The Gaussian two-peak approximation results in $\cos \theta_{1}=0.2154$ and $\cos \theta_{2}=0.2539$ for the location of the first two peaks' maxima (see Fig. 1$)^{5}$. Kinematics of the $D+D \rightarrow X+D$ reaction

\footnotetext{
${ }^{5}$ It is shown below that the Gauss distribution might arise from a sum of many near resonances. An extension of
} 
reads

$$
M_{X}^{2}=M_{D}^{2}+t+\frac{E_{1} t+P_{1} \sqrt{t\left(-4 M_{D}^{2}+t\right)} \cos \theta}{M_{D}},
$$

where $P_{1}$ is momentum of the primary deuteron, $P_{1}=8.9, E_{1}$ is its energy, and $M_{D}=1.8756 \mathrm{GeV}$. It was very unexpected to find that elastic D-D scattering gives the angle distribution with a maximum at 0.2272 , see (7.1) for $M_{X}=M_{D}$, i.e. between $\cos \theta_{1}$ and $\cos \theta_{2}$.

Similarly, elastic N-D scattering described by (7.2)

$$
M_{X}{ }^{2}=M_{N}{ }^{2}+t+1 / 2 \frac{\sqrt{P_{1}^{2}+4 M_{N}^{2}} t+P_{l} \sqrt{t\left(-4 M_{D}^{2}+t\right)} \cos \theta}{M_{D}},
$$

where $P_{1}$ is momentum of the primary neutron, $P_{1}=4.45 \mathrm{GeV}$ and $M_{X}=M_{N} \mathrm{GeV}$ has a maximum at 0.2661 , clearly shifted from the second peak location.

Thus, the explanation of the first two peaks by means of contributions of the elastic D-D and $\mathrm{N}$-D scattering fails and their origin remains unclear for the present. At first glance, the discrepancy may be attributed to systematic errors committed in the experiment, but a subsequent calculations found out that another astonishing explanation is more plausible.

To explain positions of the first two peaks, different models have been tried out. The models were based on the fact that only the recoil deuteron was unambiguously identified in [3] but masses of all other participants were unknown. Therefore, any transitions $\mathrm{X}+\mathrm{Y} \rightarrow \mathrm{Z}+\mathrm{D}$ are allowed to be taken into account. For example, a scattering $\mathrm{X}+\mathrm{D} \rightarrow \mathrm{D}+\mathrm{D}$ explains the first peak location if one assigns to $\mathrm{X}$ a value of mass of about $1913 \mathrm{Mev}$ which turns out to be close to $1916 \pm 2 \mathrm{MeV}$, observed in a pp dibaryon spectrum by Yu.A. Troyan $[4,5]$. A model $\mathrm{D}+\mathrm{D} \rightarrow \mathrm{X}+\mathrm{D}$ gives for the second peak location if one assumes $M_{X}=1965 \mathrm{MeV}$. The data from [4, 5] contain a corresponding dibaryon with $M_{X}=1965 \pm 2 \mathrm{MeV}$.

Analysis of other models showed that almost each dibaryon observed in $[4,5]$ can give a contribution to the first two peaks observed in [3], under an assumption that masses of dibaryons detected in the np-system are $1 \mathrm{MeV}$ less than the corresponding masses in the pp-system. In Table 2, considered reactions are shown in the first column. The second column specifies masses of ingoing or outgoing objects in the deuteron scattering experiment [3]. Dibaryon masses found for the pp-system in refs. $[4,5]$ are given in the third column. The reactions above the horizontal line explain the first peak and the reactions below it explain the second one. It is possible to verify that the reactions considered for explanation of the data [3] reproduce masses of all dibaryons observed in refs. [4, 5], with the exception of two of them at $2008 \pm 3$ and $2046 \pm 3 \mathrm{MeV} / \mathrm{c}^{2}$.

\section{An equidistant spectrum assumption}

With an assumption that some of dibaryons were unrecognized in the experiments $[4,5]$, it is possible to approximate the pp-dibaryon mass spectrum within rather small, at $1-2 \mathrm{MeV} / \mathrm{c}^{2}$ level, experimental errors by the formula

$$
M_{n}=M_{N N}+10.08 n,
$$

statistics may modify slightly the overall distribution. 
Table 2: Kinematically admissible masses (KAM) which might contribute to the first or second peak in the experiment [3]. Proton-proton dibaryon masses are taken from [4, 5].

\begin{tabular}{lcc}
\hline \hline Reaction & KAM & pp-dibaryon masses [4, 5] \\
\hline $\mathrm{X}+\mathrm{D} \rightarrow \mathrm{D}+\mathrm{D}$ & 1913 & $1916 \pm 2$ \\
$\mathrm{D}+\mathrm{X} \rightarrow \mathrm{D}+\mathrm{D}$ & 1884 & $1886 \pm 1$ \\
$\mathrm{D}+\mathrm{X} \rightarrow \mathrm{X}+\mathrm{D}$ & 1886 & $1886 \pm 1$ \\
$\mathrm{X}+\mathrm{X} \rightarrow \mathrm{X}+\mathrm{D}$ & 1884 & $1886 \pm 1$ \\
$\mathrm{X}+\mathrm{X} \rightarrow \mathrm{Y}+\mathrm{D}$ & $1886 \rightarrow 1898$ & $1886 \pm 1,1898 \pm 1$ \\
$\mathrm{X}+\mathrm{D} \rightarrow \mathrm{Y}+\mathrm{D}$ & $1916 \rightarrow 1884$ & $1916 \pm 2,1886 \pm 1$ \\
& $1965 \rightarrow 1937$ & $1965 \pm 2,1937 \pm 2$ \\
& $1980 \rightarrow 1953$ & $1980 \pm 2,1955 \pm 2$ \\
& $2106 \rightarrow 2086$ & $2106 \pm 2,2087 \pm 3$ \\
\hline $\mathrm{D}+\mathrm{D} \rightarrow \mathrm{X}+\mathrm{D}$ & 1965 & $1965 \pm 2$ \\
$\mathrm{X}+\mathrm{D} \rightarrow \mathrm{Y}+\mathrm{D}$ & $1886 \rightarrow 1966$ & $1886 \pm 1,1965 \pm 2$ \\
& $1898 \rightarrow 1979$ & $1898 \pm 1,1980 \pm 2$ \\
& $1916 \rightarrow 1998$ & $1916 \pm 2,1999 \pm 2$ \\
& $1937 \rightarrow 2020$ & $1937 \pm 2,2017 \pm 3$ \\
& $1999 \rightarrow 2086$ & $1999 \pm 2,2087 \pm 3$ \\
& $2017 \rightarrow 2105$ & $2017 \pm 3,2106 \pm 3$ \\
\hline \hline
\end{tabular}

where $n=0,1,2, \ldots, 40$, all values are taken in $\mathrm{MeV}, M_{N N}$ is equal to the value of mass of two protons. A quality of this assumption is seen, e.g., from a fact that only 4 dibaryons might be unrecognized in $[4,5]$ among the first 14 ones predicted by $(8.1)$.

To check the suggestion of the similarity of pp- and np-dibaryon mass spectrum, which follows from TABLE 2, we accepted the relation (8.1) for np-dibaryons too, only changing $M_{N N}$ with the deuteron value of mass. In Tables 3 and 4, the second column specifies masses of ingoing or outgoing particles, which are allowed by kinematics,

$$
M_{Y}^{2}=M_{X}^{2}+t+M_{X} P_{1} \frac{\sqrt{t\left(-4 M_{d}^{2}+t\right)}}{M_{d}^{2}} \cos \theta+\frac{M_{X} E_{1} t}{M_{d}^{2}},
$$

of the $\mathrm{X}+\mathrm{D} \rightarrow \mathrm{Y}+\mathrm{D}$ reaction. Dibaryon masses for the np-system computed according to (8.1) are shown in the third column.

One can see that each of dibaryons predicted by (8.1) in the range from 1886 to 2198 may contribute to the first or second peaks, observed in ref. [3]. Thus, new dibaryons predicted by the equidistant spectrum (8.1), taken as an assumption on basis of $[4,5]$, are also confirmed by the data [3]. Moreover, quality of the description definitely improves, since no dibaryon mass calculated using (8.1) is now lost in the description of the data from [3].

\section{The dynamical Casimir effect}

The equidistant spectrum regularity observed in [3-5] hardly can be interpreted in the frame of the 6-q bag model which predicts a different form of spectrum. One may try to assign it to some 
Table 3: Kinematically admissible masses (KAM) which might contribute to the first peak in $X+D \rightarrow Y+D$ reaction. Dibaryon masses are taken according to the equidistant spectrum assumption.

\begin{tabular}{lcc}
\hline \hline Reaction & KAM & dibaryon masses, $(8.1)$ \\
\hline $\mathrm{X}+\mathrm{D} \rightarrow \mathrm{Y}+\mathrm{D}$ & $1916 \rightarrow 1884$ & 1916,1886 \\
& $1926 \rightarrow 1895$ & 1926,1896 \\
$1936 \rightarrow 1905$ & 1936,1906 \\
$1946 \rightarrow 1916$ & 1946,1916 \\
$1956 \rightarrow 1927$ & 1956,1926 \\
$1966 \rightarrow 1938$ & 1966,1936 \\
$1976 \rightarrow 1948$ & 1976,1946 \\
$1986 \rightarrow 1959$ & 1986,1956 \\
$2047 \rightarrow 2024$ & 2047,2027 \\
$2057 \rightarrow 2034$ & 2057,2037 \\
$2067 \rightarrow 2045$ & 2067,2047 \\
$2077 \rightarrow 2056$ & 2077,2057 \\
$2087 \rightarrow 2066$ & 2087,2067 \\
$2097 \rightarrow 2078$ & 2097,2077 \\
$2107 \rightarrow 2087$ & 2107,2087 \\
$2118 \rightarrow 2099$ & 2118,2097 \\
$2128 \rightarrow 2109$ & 2128,2107 \\
$2138 \rightarrow 2120$ & 2138,2118 \\
$2148 \rightarrow 2131$ & 2148,2128 \\
$2158 \rightarrow 2141$ & 2158,2138 \\
\hline
\end{tabular}

kind of oscillator consisting of quarks coupled by gluon strings [13]. However, consideration of the oscillator wave function with the constituent quark mass value indicates that the oscillator should have enormous dimensions. For example, the state $\psi_{20}(x)$, lying in the middle of the spectrum observed in [4, 5], has the length of about $50 \mathrm{fm}$.

Actually, it was difficult to find an explanation better than to associate the spectrum with the production of pion pairs, strongly bound to compressed nucleon matter by a deep potential $-U_{0}$. The parity conservation requires pions to be produced in pairs (see below). Therefore, a value of energy of a single pion

$$
E=\sqrt{p^{2}+m^{2}-U_{0}}
$$

should be equal to $5.04 \mathrm{MeV} \equiv \mathrm{E}_{\pi}$.

A meson field in a rectangular potential well, $\varphi(\vec{r}, t)=e^{-i E t} \varphi_{E}(\vec{r})$, is described by the Klein Gordon - Fock (KGF) steady-state equation,

$$
\left.\frac{1}{r^{2}} \frac{d}{d r} r^{2} \frac{d \varphi_{E}(r)}{d r}+\left(E^{2}-m^{2}+U_{0}\right)\right) \varphi_{E}(r)=0
$$

which has a solution $\varphi_{E}(r)=A \sin p r / r$ inside the well, and $\varphi_{E}(r)=B e^{-q r} / r, q=\sqrt{m^{2}-E^{2}}$ outside it. The requirement of continuity of the logarithmic derivative at the edge of the well, $r=a$, leads to a transcendental equation

$$
p \operatorname{ctg}(p a)=\sqrt{m^{2}-E^{2}}
$$


Table 4: Kinematically admissible masses (KAM), which might contribute to the second peak in $\mathrm{X}+\mathrm{D} \rightarrow \mathrm{Y}+\mathrm{D}$ reaction. Dibaryon masses are taken according to the equidistant spectrum assumption.

\begin{tabular}{lcc}
\hline \hline Reaction & KAM & dibaryon masses, (8.1) \\
\hline $\mathrm{X}+\mathrm{D} \rightarrow \mathrm{Y}+\mathrm{D}$ & $1886 \rightarrow 1966$ & 1886,1966 \\
& $1896 \rightarrow 1977$ & 1896,1976 \\
$1916 \rightarrow 1998$ & 1916,1997 \\
$1926 \rightarrow 2009$ & 1926,2007 \\
$1936 \rightarrow 2019$ & 1936,2017 \\
$1946 \rightarrow 2030$ & 1946,2027 \\
$1997 \rightarrow 2084$ & 1997,2087 \\
$2007 \rightarrow 2095$ & 2007,2097 \\
$2017 \rightarrow 2105$ & 2017,2107 \\
$2027 \rightarrow 2116$ & 2027,2118 \\
$2037 \rightarrow 2127$ & 2037,2128 \\
$2047 \rightarrow 2137$ & 2047,2138 \\
$2057 \rightarrow 2148$ & 2057,2148 \\
$2067 \rightarrow 2158$ & 2067,2158 \\
$2077 \rightarrow 2169$ & 2077,2168 \\
$2087 \rightarrow 2179$ & 2087,2178 \\
$2097 \rightarrow 2190$ & 2097,2188 \\
$2107 \rightarrow 2200$ & 2107,2198 \\
\hline \hline
\end{tabular}

which is suitable for an estimation of relevant physical values in the interaction region. Spatial dimensions, corresponding to a given value of momentum transfer, is [14]

$$
a=\left\langle r^{2}\right\rangle^{1 / 2} \approx \sqrt{6} /|\vec{q}|=0.68 \mathrm{fm}, \quad|\vec{q}|^{2}=-t .
$$

Solving eq. (9.2) with this value of $a$, one obtains $p \approx 0.53 \mathrm{GeV}$, and using (9.1), one finds $\sqrt{U_{0}} \approx$ $0.55 \mathrm{GeV}$.

Touching dynamics of the bound pion production, we suggest that it is induced by a change of a position of walls forming the potential well, in close analogy with emission of electromagnetic waves due to a motion of resonatoršs walls. This movement is capable to give energy to the virtual pions surrounding nucleons and turn them into real particles, the bound pions. Such a mechanism is known as the dynamical Casimir effect, firstly described in [15]. It is closely connected with the Hawking radiation phenomenon and the Fulling-Unruh effect [16]. The appeal of this model is it predicts the meson field with the vacuum quantum numbers, since the mesons are produced from the vacuum state due to the strong interaction, conserving all of them. Because of this, the pion field may be present at the ground state of deuteron, as it follows from the experimental data [3], without breaking the deuteron quantum numbers. As far as the vacuum state has positive parity and the intrinsic parity of pion is negative, only even number of pions may be created in the process. Similarly, isospin conservation leads to a conclusion that pions may be produced in pairs with $I=0$, i.e. in the following vector of state:

$$
\Psi_{2 \pi}=\frac{1}{\sqrt{3}}\left(\pi_{a}^{+} \pi_{b}^{-}+\pi_{a}^{-} \pi_{b}^{+}-\pi_{a}^{0} \pi_{b}^{0}\right) .
$$


A picture of the pion production may be depicted as follows. At some instant $t_{1}$ a potential well capable to hold a bound pion energy level of a value $\varepsilon$ is formed. Then, rather quickly, the energy level $\mathrm{E}_{\pi}>\varepsilon$ is developed due to a shrinkage of the potential well in the nucleon collision process. After that at moment $t_{2}$, when nucleons is moving away, the energy level returns to the value $\varepsilon$, and afterwards it changes again to the Yukawa vacuum, corresponding $E=0$ and $q=m$. From mathematical viewpoint, creation of bound pions in this framework is totally equivalent to the parametric excitation of the quantum oscillator which appears after the quantization of the field.

\section{Pion Bose-Einstein condensate}

The time dependent KGF equation,

$$
\left[\frac{\partial^{2}}{\partial t^{2}}-\frac{\partial^{2}}{\partial r^{2}}+m^{2}-U_{0}\right] \psi(r, t)=0,
$$

with the evolving boundary conditions gives the wave function inside the well,

$$
\varphi(r, t)=\chi(t) \sin p r / r
$$

where $\chi(t)$ describes an increasing amplitude of the field which manifests itself in the pion production. It obeys the equation

$$
\frac{\partial^{2} \chi(t)}{\partial t^{2}}+\left(p^{2}+m^{2}-U_{0}\right) \chi(t)=0
$$

which has the same form as one for a classical oscillator with the varying frequency $\omega(t)=E(t)$. Therefore, it is possible to introduce the oscillator Hamiltonian

$$
H=\frac{1}{2}\left(\pi_{\omega}^{2}+\omega^{2}(t) \chi_{\omega}^{2}\right)=\omega(t)\left(a_{\omega}^{+}(t) a_{\omega}(t)+\frac{1}{2}\right)
$$

and draw eq. (10.2) in the Hamiltonian formalism framework:

$$
\frac{\partial H}{\partial \pi_{\omega}}=\dot{\chi}_{\omega}, \quad-\frac{\partial H}{\partial \chi_{\omega}}=\dot{\pi}_{\omega},
$$

where

$$
\chi_{\omega}=\frac{a_{\omega}+a_{\omega}^{+}}{\sqrt{2 \omega}}, \quad \pi_{\omega}=\frac{a_{\omega}-a_{\omega}^{+}}{\sqrt{2 \omega}} .
$$

The quantization may be performed by analogy with the similar procedure for a quantum field in the box via replacing functions $a_{\omega}(t)$ and $a_{\omega}^{+}(t)$ by the corresponding operators. The only non-essential difference is that now the field does not vanish at the boundary, but terminates in an exponentially decaying tail outside the potential well. Fields of this type are met in solid-state physics [17]. Thus, the quantized field in the Heisenberg picture is written as

$$
\hat{\varphi}(r, t)=\hat{\chi}_{\omega}(t) \sin p r / r=\left(\frac{\hat{a}_{\omega}^{\dagger}(t)+\hat{a}_{\omega}(t)}{\sqrt{2 \omega_{1}}}\right) \sin p r / r,
$$


for any $t$ in the range of the pion production, $t_{1} \leq t \leq t_{2}$. Here $\omega_{1}=\omega\left(t_{1}\right)=\varepsilon$. The time evolution of the field may be expressed in an equivalent form, using Bogoliubov's canonical transformation (BCT):

$$
\left(\begin{array}{c}
\hat{a}(\Delta t) \\
\hat{a}^{+}(\Delta t)
\end{array}\right)=\overbrace{\left(\begin{array}{cc}
u(\Delta t) & v(\Delta t) \\
u^{*}(\Delta t) & v^{*}(\Delta t)
\end{array}\right)}^{S(\Delta t)}\left(\begin{array}{c}
\hat{a}_{S} \\
\hat{a}_{S}^{+}
\end{array}\right),
$$

where $\hat{a}_{S}, \hat{a}_{S}^{+}$are the annihilation and production operators in the Schrödinger representation, $u(\Delta t)$ and $v(\Delta t)$ are usual (non-operator) functions. It is obvious that matrices $S(\Delta t)$ generate a group under multiplication,

$$
S(\Delta t) \equiv S\left(\Delta t_{1}+\ldots+\Delta t_{n}\right)=S\left(\Delta t_{n}\right) \ldots S\left(\Delta t_{1}\right) .
$$

The commutation relation requirement $\left[\hat{a}(t), \hat{a}^{+}(t)\right]=1$ leads to a constraint

$$
|u(t)|^{2}-|v(t)|^{2}=1
$$

which means that the group of dynamical symmetry is $S U(1,1)$.

Now we turn to the Schrödinger picture and define the group action in the space of state vectors, rather than in a space of the parameters describing evolution of operators. Lie algebra of $S U(1,1)$ is defined by the commutation relations

$$
\left[\hat{K}_{1}, \hat{K}_{2}\right]=-i \hat{K}_{0},\left[\hat{K}_{2}, \hat{K}_{0}\right]=i \hat{K}_{1}, \quad\left[\hat{K}_{0}, \hat{K}_{1}\right]=i \hat{K}_{2}
$$

or, after introducing

$$
\hat{K}_{ \pm}= \pm i\left(\hat{K}_{1} \pm i \hat{K}_{2}\right)
$$

by

$$
\left[\hat{K}_{0}, \hat{K}_{ \pm}\right]= \pm \hat{K}_{ \pm}, \quad\left[\hat{K}_{-}, \hat{K}_{+}\right]=2 \hat{K}_{0}
$$

One can express elements of the $S U(1,1)$ group through its generators:

$$
\hat{S}(d t)=e^{\left(\beta \hat{K}_{+}-\beta^{*} \hat{K}_{-}-i \gamma \hat{K}_{0}\right) d t} .
$$

But in the case of the Hamiltonian evolution

$$
\hat{S}(d t)=e^{-i \hat{H} d t}
$$

so that it is possible to rewrite Hamiltonian (10.3) in the form

$$
\hat{H}=i\left(\beta \hat{K}_{+}-\beta^{*} \hat{K}_{-}-i \gamma \hat{K}_{0}\right) .
$$

Corresponding expressions for $\hat{K}_{+}, \hat{K}_{-}$and $\hat{K}_{0}$ are

$$
\hat{K}_{+}=\frac{\left(\hat{a}^{\dagger}\right)^{2}}{2}, \hat{K}_{-}=\frac{\hat{a}^{2}}{2}, \hat{K}_{0}=\frac{\hat{a} \hat{a}^{\dagger}+\hat{a}^{\dagger} \hat{a}}{4}
$$

for $\pi^{0} \pi^{0}$ and

$$
\hat{K}_{+}=\hat{a}_{+}^{\dagger} \hat{a}_{-}^{\dagger}, \hat{K}_{-}=\hat{a}_{+} \hat{a}_{-}, \hat{K}_{0}=\frac{1}{2}\left(\hat{a}_{+}^{\dagger} \hat{a}_{+}+\hat{a}_{-}^{\dagger} \hat{a}_{-}+1\right)
$$


for $\pi^{+} \pi^{-}$. In fact, the operators $\hat{K}_{0}$ do not lead to a change of a particle number and it is possible to omit them, at least for particle number distribution calculations. Thus, the evolution operator may be defined as an element of the $S U(1,1)$ group of a kind $\hat{S}(t)=\exp \left(\xi \hat{K}_{+}-\xi^{*} \hat{K}_{-}\right)$. Therefore, the state of system at moment $t$ is estimated as

$$
\left|\psi_{t}\right\rangle=\exp \left(\xi \hat{K}_{+}-\xi^{*} \hat{K}_{-}\right)|0\rangle
$$

It is possible to notice a similarity of this state to the Glauber coherent state [18]

$$
\left|\psi_{G}\right\rangle=e^{\alpha a^{\dagger}-\alpha^{*} a}|0\rangle=e^{-|\alpha|^{2} / 2} \sum_{n=0}^{\infty} \frac{\alpha^{n}}{\sqrt{n !}}|n\rangle
$$

which leads to the Poisson distribution for the probability to find $n$ particles in the $\left|\psi_{G}\right\rangle$ state,

$$
w_{n}=\left|\left\langle n \mid \psi_{G}\right\rangle\right|^{2}=e^{-|\alpha|^{2}} \frac{|\alpha|^{2 n}}{n !},\langle n\rangle=|\alpha|^{2} .
$$

Similarly, the state $\left|\psi_{t}\right\rangle$ reads [19]

$$
\left|\psi_{t}\right\rangle=\left(1-|\eta|^{2}\right)^{k} \sum_{m=0}^{\infty}\left(\frac{\Gamma(m+2 k)}{m ! \Gamma(2 k)}\right)^{1 / 2} \eta^{m}|k, k+m\rangle .
$$

Here $k$ describes a representations of $S U(1,1), k=1 / 4$ for $\pi^{0} \pi^{0}$ and $k=\frac{1}{2}$ for $\pi^{+} \pi^{-}, m$ is a number of pion pairs created, $\eta=\sqrt{\rho} e^{i \varphi}$. A value of $\rho$ may be expressed through the coefficients $u\left(t_{2}\right)$ and $v\left(t_{2}\right)$ of BCT at the end of the pion production, $\rho=|v|^{2} /|u|^{2}$, and $e^{i \varphi}$ is a phase factor, unessential here. The probability to find $n=2 m$ particles in the state is equal to

$$
w_{n}=\left|\left\langle n \mid \psi_{t}\right\rangle\right|^{2}=\sqrt{1-\rho} \frac{n !}{2^{n}[(n / 2) !]^{2}} \rho^{n / 2},
$$

for $\pi^{0} \pi^{0}$ system. For $\pi^{+} \pi^{-}$, it is

$$
w_{n}=\left|\left\langle n \mid \psi_{t}\right\rangle\right|^{2}=(1-\rho) \rho^{n / 2}
$$

\section{Calculation of $\rho$}

The model under consideration allows to find an exact solution. To arrive at it, one should only calculate a value of $\rho$. This can be done in the framework of a certain scattering problem for a quantum mechanical particle [20,21], if we accept the usual scattering matrix formalism assumption: $t_{1} \rightarrow-\infty$ and $t_{2} \rightarrow+\infty$.

In order to make sure of that, let us come back to the Bogoliubov transformation (10.4). One can see that the coefficients $u(t)$ and $v(t)$ should satisfy eq. (10.2), because the field should satisfy eq. (10.1), taken in the operator form. Boundary conditions for the appropriate solutions of (10.2) follow from requirements

$$
\begin{aligned}
& \hat{a}(t) \rightarrow \exp \left(i \omega_{1} t\right) \hat{a}_{S}, \quad t \rightarrow-\infty \\
& \hat{a}(t) \rightarrow C_{1} \exp \left(i \omega_{1} t\right) \hat{a}_{S}+C_{2} \exp \left(i \omega_{1} t\right) \hat{a}_{S}^{\dagger}, \quad t \rightarrow+\infty .
\end{aligned}
$$


Here the annihilation operator for the outgoing field is taken in the most general form consistent with its $\exp \left(i \omega_{1} t\right)$ time dependence and the ingoing field operator describes the state without pions. This implies

$$
\begin{aligned}
u(t) & \rightarrow \exp \left(i \omega_{1} t\right), \quad v(t) \rightarrow 0, \quad t \rightarrow-\infty, \\
u(t) \rightarrow C_{1} \exp \left(i \omega_{1} t\right), \quad v(t) & \rightarrow C_{2} \exp \left(i \omega_{1} t\right), \quad t \rightarrow+\infty .
\end{aligned}
$$

Thus, the unknown parameter $\rho$ may be written as

$$
\rho\left(t_{2}\right)=\frac{\left|v\left(t_{2}\right)\right|^{2}}{\left|u\left(t_{2}\right)\right|^{2}}=\frac{\left|C_{2}\right|^{2}}{\left|C_{1}\right|^{2}} .
$$

The requirement (10.5) means that $\left|C_{1}\right|^{2}$ and $\left|C_{2}\right|^{2}$ are not independent. This gives

$$
\left|C_{1}\right|^{2}=\frac{1}{1-\rho}, \quad\left|C_{2}\right|^{2}=\frac{\rho}{1-\rho} .
$$

A variable

$$
w(t)=\left(u(t)+v(t)^{*}\right) / C_{1}
$$

also satisfies (10.2) together with boundary conditions

$$
w(t) \rightarrow e^{i \omega_{1} t} / C_{1}, t \rightarrow-\infty ; \quad w(t) \rightarrow e^{i \omega_{1} t}+\frac{C_{2}^{*}}{C_{1}} e^{-i \omega_{1} t}, t \rightarrow+\infty .
$$

There is a close analogy between eq. (10.2) for $w(t)$, and its solution (11.1), and the Schrödinger equation

$$
\frac{\partial^{2} \psi(x)}{\partial x^{2}}+\left(\frac{k^{2}}{2 m}-V(x)\right) \psi(x)=0,
$$

corresponding to the scattering problem of a particle by a potential $V(x)$, which has a solution [9]

$$
\mathrm{e}^{\mathrm{i} \mathrm{k}_{1} x}+B \mathrm{e}^{-\mathrm{i} \mathrm{k}_{1} x}
$$

in the region containing the incident and the scattered wave. In this framework, the value of $\rho$ corresponds to the reflection coefficient, $\rho=R$, of the scattering problem. To achieve the total mathematical equivalence of the both models, it is necessary to replace $2 m$ by 1 in the Schrödinger equation, to transpose ingoing and outgoing states, and to map:

$$
t \leftrightarrow x, \quad E^{2}(t)-V(t) \leftrightarrow k^{2}(x)-V(x),
$$

where a time-dependent potential $V(t)$ simulates the changing boundary conditions. In a simple case when

$$
E(t)=\left\{\begin{array}{cl}
\mathrm{E}_{\pi}=5.04 \mathrm{MeV}, & \text { for } 0<t<\tau, \\
\varepsilon, & \text { for } 0>t, \text { or } t>\tau,
\end{array}\right.
$$

one has the scattering by a rectangular potential well of a depth

$$
V_{0}=\mathrm{E}_{\pi}^{2}-\varepsilon^{2}
$$


Subject to this proviso, it is possible to find:

$$
\rho=\frac{1}{1+\delta^{2}}, \quad \delta=\frac{2 \varepsilon \mathrm{E}_{\pi}}{V_{0} \sin \mathrm{E}_{\pi} \tau}
$$

where $\tau \sim 1 / \Gamma, \Gamma$ is the dibaryon width, $\varepsilon$ is the only unknown parameter which can be found in further experiments. The data accuracy in $[4,5]$ does not permit to estimate $\varepsilon$ but it allows to conclude that $\rho$ is very close to 1 , see (10.8) for the registered value of $n=80$. The distribution (10.7) rapidly decreases with $n$ therefore only the bound $\pi^{+} \pi^{-}$pairs contribute to the heavy dibaryon tail observed in $[4,5]$.

\section{Discussion and Conclusions}

In the present paper, we confine ourself to consideration of some experimental evidences for MB production with $\mathrm{B}=2$, leaving aside a possibility of observation of tribaryons, tetrabaryons, pentabaryons, etc. One may wonder, why so few if any signs of dibaryons exist currently. And particularly, why the partial-wave analysis (PWA) of N-N elastic scattering did not reveal them. There are at least two reasonable responses to the second question. First of all, data reported by WASA-at-COSY Collaboration [11] if they really inform about the dibaryon natural occurrence mean that a precision of PWA remains unsatisfactory yet. The second explanation might be based on a suggestion that some dibaryons in intermediate states of the elastic N-N scattering may appear near their mass shell only if they are escorted by pions. Corresponding intermediate states provide therefore the elastic scattering amplitude $\mathrm{NN} \rightarrow$ dibaryon $+\mathrm{n} \pi \rightarrow \mathrm{NN}$ with a cut instead a pole which is usually looked for in PWA. Our suggestion may be grounded in part by the following reasoning. All dibaryons reported in $[4,5]$ were observed in inelastic N-N interactions with additional secondary pions. The elastic N-N scattering amplitude is connected with the inelastic N-N interactions by the unitarity condition which provides it with all possible intermediate states. The extra pions take away an excess of excitation energy - a process which is a some kind of annealing. This may reconcile two opposite requirements imposed simultaneously on the system: it must be strongly compressed to form a compound state and it must be cold enough, since highly excited levels are usually short-living and elusive.

The second natural question concerns calculations of NN-interactions below the one-pion threshold in the Chiral Perturbation Theory (ChPT) framework. Why were there no dibaryons? The dibaryon with $M=2.37 \mathrm{GeV}$ stand above one-pion threshold and therefore off this discussion. As regards light dibaryons, it follows from (5) that a necessary condition for their existence is $m_{\pi}>0$. At first sight, this possibility may be considered in ChPT with the explicit symmetry breaking. Nevertheless, it is impossible. As it is argued above, the light dibaryons are an experimental evidence for the pion Bose-Einstein condensate appearance. It is a purely nonperturbative effect described by Bogoliubov's transformation which produces a pion state beyond the range of the Fock space. Perhaps one can find some traces of this state in ChPT known there as contact terms. Sometimes they are interpreted as an evidence for the existence of the NN-dibaryon vertex, see, e.g., [22]. These terms are introduced if one should describe short-range interactions where a 
value of parameter $Q / \Lambda_{\chi}$ is large and the ChPT series is badly convergent. J. Soto and J. Tarrús used the same method for a low energy effective field approximation of QCD for an explanation of the nucleon-nucleon scattering amplitudes and obtained an excellent descriptions of the phase shifts [23].

All lattice QCD collaborations have found stable NN-dibaryons and dibaryons containing squarks, but quark masses in their calculations are higher than the physical values, see, e.g., [24, 25]. Chiral extrapolations of these results to the physical point gave, however, evidences against the existence of such dibaryons, see, e.g., [26]. These calculations deal with ground states and say nothing about unstable states corresponding to a possibility of two-baryon fusion into 6-quark bag with a value of mass larger than a sum of masses of the initial baryons. Recent progress in excited baryon spectroscopy is depicted in [27, 28]. Corresponding results based on nonphysical quark masses too cover only one-baryon states so far and are in a poor agreement with experimental $\mathrm{N}$ and $\Delta$ excitation spectra. The first excited state in two-nucleon system was found in lattice QCD in [29] but with a heavy quark mass corresponding to $m_{\pi}=0.8 \mathrm{GeV}$. Therefore, predicting quasibound states of a multibaryon systems remains a difficult challenge in lattice QCD till now.

In a paper B.M.Abramov et al [30], an opinion that Troyan's resonances were only fluctuations of background was expressed. In practice, substraction of a background requires a design of special models, and Yu.A. Troyan elaborated one described in $[4,5]$. We do not know any explicit objections against his method, while the solid line in the main figure of the paper [30] is only an optimal approximation of the experimental invariant mass spectrum containing, in the general case, a sum of background and dibaryon contributions. Therefore, this line cannot be interpreted as the background. It could not be considered as well as a proof of dibaryon absence by reason of its smoothness, since usage of more delicate approximations of the experimental data would reveal a presence of peaks in the spectrum. Moreover, it is impossible to interpret as statistical fluctuations peaks shown in Fig. 1 in the paper of Yu.A. Troyan. Indeed, statistical fluctuations in one cell of a histogram are Poisson ones. Therefore, their standard deviation should be equal to $\sqrt{N}$, where $N$ is a number of events per a cell, shown in Y-axis in the figure. It is readily checkable that the fluctuations near the peak of the histogram overtop substantially the suggested value. More accurate study of fluctuations with taking into account experimental errors were performed by Yu.A. Troyan in [5]. He showed that average error of $M_{p p}$ not far from the beginning of the spectrum is about 2.4 MeV. This is quite enough for recognition of isolated dibaryons which are separated from each other by a distance of $10 \mathrm{Mev}$. However, mean correlation distance $L_{c}=\Gamma / 2$, of the fluctuations identified as dibaryons at small values of $M_{p p}$ is of the same order. This implies that the true resonant widths of the dibaryons should might be less than those seen in Fig. 1 in [5] and, actually, the peaks might be higher than they appear in the figure. Therefore, very small probabilities of the dibaryons might be a maverick, found in [5], seem to be rather realistic. To confirm this suggestion future experiments must have resolution at least at a level $1 \mathrm{MeV}$ due to higher statistics and less experimental errors.

There is another reason might explain the difference between Yu.A. Troyan and B.M. Abramov et al experiments. As it was suggested in our paper, observation of dibaryons is possible only under the conditions of "deep cooling". Let us compare. Only a reaction $p n \rightarrow p p \pi^{-}$was considered in the paper of B.M.Abramov et al. Reactions investigated by Yu.A. Troyan include: $p n \rightarrow p p \pi^{-}$, $p n \rightarrow p p \pi^{-} \pi^{0}, p n \rightarrow p p \pi^{+} \pi^{-} \pi^{-}, p n \rightarrow p p \pi^{+} \pi^{-} \pi^{-} \pi^{0}$. We can see from kinematics, and explicit 
comparison of the data from [4, 5] and [30], that the effective mass spectrum is hotter indeed in Abramov's experiment. The Bose-Einstein condensate may not arise at such conditions. Therefore, one might suggest that the first reaction from the Troyan's list gave only a noise to the dibaryon signal observed. And we see, indeed, that the tail of distribution in Fig.1 in the paper of Yu.A. Troyan $[4,5]$ contains visible strips in which the fluctuations are symmetrical against the background. This may be a signature of a small dibaryon contribution in this region.

Our consideration of the data on the hard deuteron-deuteron scattering [3] meets the expectation to observe the transition of nucleon matter into other states using the method of deep cooling which allows to recognize quasi-resonance peaks in the reaction cross-section. As concerns the dibaryons obeying the equidistant spectrum regularity observed in [3-5], they hardly can be interpreted in the frame of the 6-q bag model. It is very likely to assign them to the production of pion pairs strongly bound to compressed nucleon matter. The analysis of the data from [3] reveals the possibility of presence of the pion Bose-Einstein condensate in the ground state of deuteron, see (10.6). According to this analysis, the condensed pion field in deuteron can change in hard nuclear collisions. The pion Bose-Einstein condensate might also appear in the compressed proton-proton system subjected to a proper cooling, according to the experimental hints from $[4,5]$. The theory predicts the characteristic mass distribution for dibaryons of this type, which may be considered as an experimentally feasible signature of the pion Bose-Einstein condensate. Further experimental studies based on modern experimental equipment and more abundant statistics are necessary to verify the conclusions of our paper. Experiments [3] and [4, 5] allow to hope that these efforts will not be unavailing.

It is reasonable to ask whether the pion Bose-Einstein condensate arises in compressed $k$ nucleon systems for $k>2$. If this is true, it can impact essentially on collective flows at the final stage of high-energy nuclear collisions, especially on the sideflow [31].

It should be noted that the state of pion field (10.6) has a mathematical and physical prototype in quantum optics, known there as the squeezed vacuum [32]. Using this interpretation, one may qualify the operator $\hat{S}(t)=\exp \left(\xi \hat{K}_{+}-\xi^{*} \hat{K}_{-}\right)$defined above as the squeeze operator. An appropriate squeeze factor $r$ can be expressed through the expectation value of the pion number in this state: $\sinh ^{2} r=\left\langle\hat{a}^{\dagger} \hat{a}\right\rangle$ for $\pi^{0} \pi^{0}$ and $\sinh ^{2} r=\left\langle\hat{a}_{ \pm}^{\dagger} \hat{a}_{ \pm}\right\rangle$for $\pi^{+} \pi^{-}$pairs.

Appreciations. We are grateful to N.B. Bogdanova and A.P. Ierusalimov for useful software support [33, 34] of our investigation.

\section{References}

[1] B.F. Kostenko, J. Pribiš, Yad. Fiz. 75, 888 (2012).

[2] B.F. Kostenko, J. Pribiš, and V. Filinova, PoS (Baldin ISHEPP XXI) 105.

[3] A.M. Baldin et al., Differential Elastic Proton-Proton, Nucleon-Deuteron and Deuteron-Deuteron Scatterings at Big Transfer Momenta, JINR Communication 1-12397, Dubna, 1979 (in Russian).

[4] Yu.A. Troyan, V.N. Pechenov, Yad. Fiz. 56, 201 (1993).

[5] Yu.A. Troyan, Fiz. Elem. Chastits At. Yadra 24, 683 (1993).

[6] V. Borka Jovanović, S. R. Ignjatović, D. Borka, and P. Jovanović, Phys. Rev. D 82, 117501 (2010).

[7] J. Beringer et al. (Particle Data Group), Phys. Rev. D 86, 010001 (2012). 
[8] R.H. Dalitz, L.J. Reiners, in Hadron Structure as Known from Electromagnetic and Strong Interactions, Proc. of the Hadron ' 77 Conference, Veda, 1977, p. 11.

[9] L.D. Landau, E.M. Lifshitz. Quantum Mechanics, Oxford, Pergamon, 1987.

[10] H.M. Pilkuhn. Relativistic Particle Physics, New York, Springer, 1979.

[11] P. Adlarson et al. (WASA-at-COSY Collaboration), Phys. Rev. Lett. 106, 242302 (2011).

[12] Yu.A. Troyan et al., Yad. Fiz. 63, 1648 (2000).

[13] E. Wang, C.W. Wong, Il Nuov. Cimento, 86A (1985)283.

[14] F. Halzen, A.D. Martin. Quarks and Leptons. New York, John Wiley, 1984, Chap. 8.2.

[15] S.A. Fulling, P.C.W. Davies, Proc. R. Soc. Lond. A, 348 (1976) 393.

[16] N.D. Birrell, P.C.W. Davies. Quantum Fields in Curved Space. Cambridge, Cabridge University Press, 1982.

[17] W. Shockley, Phys. Rev. 56 (1939) 317.

[18] R.J. Glauber, Phys. Rev. 130, 2529 (1963); 131, 2766 (1963).

[19] A. Perelomov, Generalized Coherent States and Their Applications, Springer, 1986.

[20] A.M. Dykhne, JETP 38, 570 (1960).

[21] V.S. Popov, A.M. Perelomov, JETP 56, 1375 (1969).

[22] Shung-ichi Ando, Eur. Phys. J. A33, 185(2007).

[23] J. Soto and J. Tarrús, Phys. Rev. C 78, 024003 (2008).

[24] Takashi Inoue (HAL QCD Collaboration), Lattice 2011, arXiv:1111.5098

[25] S.R. Beane et al. (NPLQCD Collaboration), Phys. Rev. Lett. 106, 162001 (2011).

[26] P.E. Shanahan, A.W. Thomas, R.D. Young, arXiv:1308.1748

[27] Huey-Wen Lin, Chinese Jour. Phys. 49, 827 (2011).

[28] R.G. Edwards, N. Mathur, D.G. Richards, S.J. Wallace, Phys Rev D 87, 054506 (2013).

[29] T. Yamazaki, Y. Kuramashi, A. Ukawa, Phys. Rev. D 84054506 (2011).

[30] B.M.Abramov et al. Z.Phys. C 69, 409-413 (1996).

[31] N. Herrmann, J.P. Wessels, and T. Wienold, Annu. Rev. Nucl. Sci. 49, 581 (1999).

[32] D.F. Walls, G.J. Milburn. Quantum Optics, Berlin, Springer, 2008.

[33] N.B. Bogdanova. Orthonormal Polynomial Expantion Method with Errors in Variables. JINR Communication, E11-98-3, 1998.

[34] A.P. Ierusalimov et al, System of Graphics Digitization, JINR Rapid Communication, No.6[86]-97, pp.98-102, 1997. 